\title{
Simulation of Human Balance Impairment on a Biped Robot
}

\author{
Abdalla B. Zreiba' ${ }^{1}$, Mojtaba Ahmadi ${ }^{1}$, Ali Etemad ${ }^{2}$ \\ ${ }^{1}$ Carleton University/Mechanical and Aerospace Engineering Department \\ abdullahzreiba@cmail.carleton.ca; mojtabaahmadi@cunet.carleton.ca \\ 2Queen's University/ Department of Electrical and Computer Engineering \\ 99 University Avenue Drive, Kingston, Canada \\ ali.etemad@queensu.ca
}

\section{Extended Abstract}

When an assistive device is attached to a human with changing behaviour (for example a person with compromised balance), then the question is how the assistive device adapts to that person, and how to detect which state the person is in. This paper aims to develop an adaptive learning controller to control assistive devices. The controller monitors human interactions, pre-processes human states, classifies its behaviors, and based on a learning algorithm, selects various types of controllers or parameters to optimize the assistance provided to the human user for its varying behaviors or conditions. The application of this research project will be in the enhancement of human balance. In particular, the methodology will be adapted to the 2D dynamic biped. The adaptive controller will assist in detecting and preventing falls due to balance impairment.

Human behaviors are complicated and an intelligent controller needs to be adaptive to these behaviours, which can help them to regain function. Maintaining balance depends on the actions of the human, and this question remains to be investigated with more subjects and patients. Using a dynamic biped to simulate a patient attached with an assistive device receiving locomotion behaviors information, the classifier identifies the biped states, and based on a learned controller takes the right control behaviour or strategy to adjust the assistance provided to the biped.

The objective of the simulation of human balance impairment on a biped robot is to learn which behaviour can balance the biped (human) when compromising behaviour has occurs. In other words, to determine whether the biped is going to fall or not, a classification approach should be used. A reduced state space (composed of motion measurements and stability relevant parameters) is explored for the impairment classification on the biped. Once the compromising behaviour is identified, a Q-learning algorithm will decide which actions or control behaviours strategies should be used for this application.

The adaptive controller was tested in simulation on a 7- DOF planar biped simulation. While the biped is generating stable periodic walking patterns, impairment composed of lowering the joint torque of one of legs of the biped is introduced, causing the reference COG to diverge from the reference CMP, resulting in biped falling. Simulations show that when the same walking process is repeated using the learned algorithm, it accumulates the positive and the negative rewards and the adaptive controller is able to identify the impairment and activate a new successful strategy. The impairments as well as the successful strategy are kept in the learner's memory via the Q-learning algorithm, allowing the controller to select the same strategy when it is faced with new similar impairments in the future. 